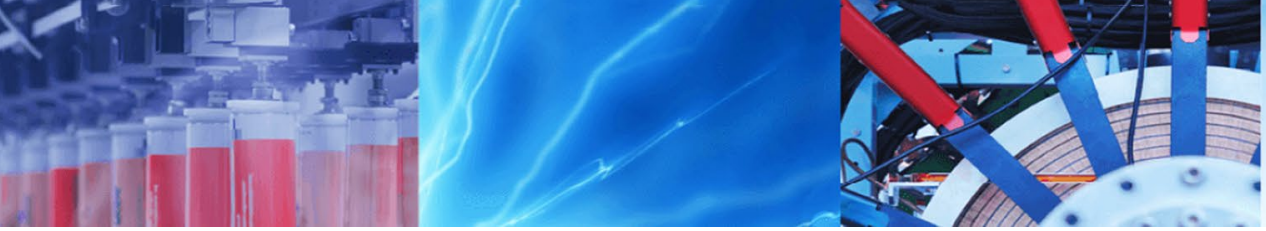

Research Article

\title{
Quality and robustness optimization design method for electromagnetic devices consider manufacturing uncertainties and working point migration of permanent magnets
}

\author{
Xuerong Ye ${ }^{1} \cdot$ Hao Chen ${ }^{1} \cdot$ Cen Chen ${ }^{1} \cdot$ Guofu Zhai $^{1}$
}

Received: 8 January 2020 / Accepted: 6 March 2020 / Published online: 20 March 2020

(c) Springer Nature Switzerland AG 2020

\begin{abstract}
Electromagnetic devices (EMDs) containing permanent magnets are common devices showing low power con-sumption, small size, and high sensitivity. In batch production of EMDs there is a robustness problem linked to the migration of the working point of permanent magnets and to their manufacturing uncertainties. In turn, the design optimization for EMDs has attracted large attention. Robust design optimization (RDO) is one of the most effective methods to boost quality and robustness of EMDs. However, state-of-art RDO methods ignore the various uncertainties from manufacture processing, production environment, and working point migration of permanent magnets. To address those problems, this study presents an efficient and universal method based on the working point migration and various uncertainties of output characteristics for electromagnetic devices. At first, we introduce a two-dimensional factor design scheme considering the working point migration and manufacturing conditions. Then, a robustness assessment measure suitable for different optimization types is proposed. On these bases, the optimization model is established, considering the permanent working point migration for magnets, manufacturing tolerances and robustness constraints, and a particle swarm algorithm is used to obtain the solution. Finally, the effectiveness of the method is verified by a case study involving a specific type of electromagnetic device with permanent magnets.
\end{abstract}

Keywords Robust optimization design - Manufacture processing - Uncertainties - Working point migration . Electromagnetic devices · Permanent magnets

\section{Introduction}

Electromagnetic devices (EMDs) are widely used for photovoltaic power generation, wind power generation, electric vehicles and charging stations (the main application of electric vehicles is shown in Fig. 1, which plays a role in energy conversion, signal control, safety protection, etc.) [1-3]. The operating characteristics of EMDs, their quality and their robustness in turn determine the performance and reliability of the whole electrical control systems. In recent years, electrical control systems have developed in the direction of long-life, high reliability, miniaturization and low power consumption, which sets higher demands for the power consumption, volume, robustness and sensitivity of EMDs [4-6]. EMDs with permanent magnets are suitable to achieve the above goals and are thus extensively used in devices like relays, motors, actuators, and sensors [7-9]. At the same time, the extensive use of permanent magnets poses novel challenges related to the reliability and robustness of EMDs. On the one hand, the uncertainty of the manufacturing is increased. On the other hand, the working point migration of permanent magnets during the cycle of operation enhances the challenges to the quality and robustness of EMDs. In

$\triangle$ Cen Chen, hao.hitee@gmail.com | 'School of Electrical Engineering and Automation, Harbin Institute of Technology, 92 West Dazhi Street, Nan Gang District, Harbin 150001, People's Republic of China. 


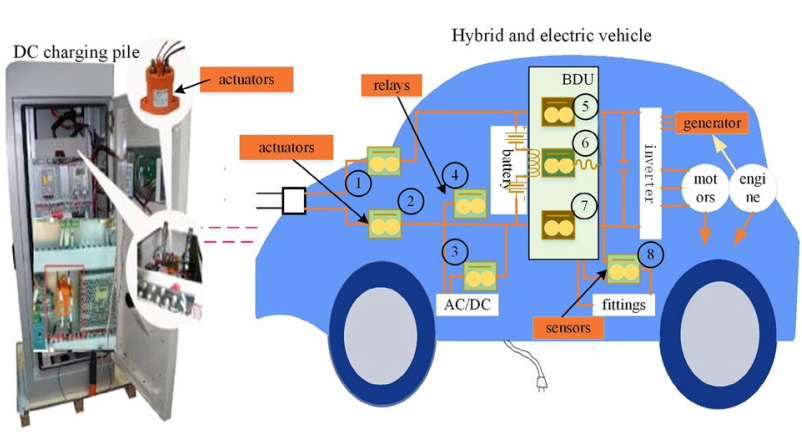

Fig. 1 Application of electromagnetic devices in charging piles and electric vehicles

turn, there is an increasing concern about the quality and robustness optimization design of EMDs [10-13].

EMDs quality and robustness are affected by various uncertainties, including material properties, assembly errors, and working point migration of permanent magnets. Indeed, EMDs with permanent magnets are not simple devices for what concern the handling of the working point migration and the manufacturing uncertainties. Finite Element Method (FEM) is a commonly used method for the analysis of permanent magnet working point migration. However, FEM only serves to analyze the working point migration and does not apply to the design of EMDs [14, 15]. In order to employ working point migration models to the optimization of EMDs design process, several approaches have been proposed [16-18]. Nonetheless, state-of-art methods consider only the influence of the permanent magnet working point migration on the output characteristics of the EMDs, without consider the robustness of the EMDs against various uncertainties from manufacture processing, operation environment engender working point migration of permanent magnets.

Subsequently, several optimization methods to increase the robustness of EMDs have been proposed. They may be divided in two main categories: (1) methods based on direct optimization of robustness, such as the Design of Experiments and the Taguchi robustness optimization method. Taguchi [19] introduced the quality loss theory into the product design and manufacturing process for establishing the tolerance design model, and took the output characteristic and its fluctuation as the basis for calculating the cost loss [20-22]. Later, scholars combined the cost and performance to establish the comprehensive quality loss function [23-25]. The abovementioned methods are suitable for the tolerance design of electronic system, since it is easy to estimate the cost of each component. And (2) methods based on indirect optimization of approximation models by using different kinds of optimization algorithms, including intelligent algorithms [26-31]. Although these methods may theoretically improve the robustness of the EMDs, they fail to effectively consider the impact of permanent magnet working point migration, such that the batch production consistency has not been greatly improved. In addition those methods neglect the "calculation catastrophe" caused by different types in robustness optimization design process.

In order to avoid the above problems, robustness design optimization should take into account the batch production and operation conditions. To this aim, a new approach for electromagnetic device robust design considering the working point migration of permanent magnets is proposed in this study. In particular, our approach carefully considers the manufacturing and permanent magnet working point migration factors. At first, we analyze the interactions among the key parameters of EMDs in order to reduce the number of models and to enhance the model accuracy, while avoiding the influence of the robustness design and optimization. Then, based on the traditional Taguchi parameter design method, a twodimensional uncertainties scheme is developed by considering the permanent magnet working point migration as well as the manufacturing uncertainties. Moreover, a high-order response surface approximation model of the EMDs, including the output mean and variance, is built to improve the approximate modelling accuracy and efficiency of the electromagnetic devices. Finally, a robustness assessment measure suitable for different optimization types is designed to address the problem of local optimization in the Taguchi method and to improve the optimization efficiency to some extent. To prove the effectiveness of the proposed method we present the robust optimization of a typical electromagnetic device with permanent magnets.

\section{The optimization method proposed in this study}

\subsection{Overall flow of the optimization method}

The design parameters have been selected according to the research object and the robustness optimization target. The various uncertainties from the manufacturing process and the operation environment have been determined by the current state of art of the manufacturing process, whereas the permanent magnet working point of the EMDs can be calculated from the load condition. Then, the sample points (a series of virtual prototypes of EMDs) within the allowed range of the design parameters of the EMDs have been generated by the two-dimensional uncertainties scheme, which effectively considers the working point migration of the permanent magnets and the batch production manufacturing conditions in 
the robustness of the design processes. Furthermore, to improve the accuracy of the approximate model and the optimization effect of the robustness design, the correlations between the design parameters have been evaluated, only retaining the cross terms between strongly correlated parameters.

Using of sample points and the correlation terms between design parameters, the output characteristics of each prototype have been obtained by the finite element method. Then, the high-order response surface model is adopted to evaluate the relationship between each design parameter and the output characteristics of the electromagnetic devices, including the output mean and variance. Finally, we have designed a robustness figure of merit that considers the robustness of EMDs to measure the output performance robustness of various types of EMDs. In this way, we solve the problem of local optimization in the Taguchi method and also improve the optimization efficiency, while a qualified rate of production. One the robustness optimization model is established, we employ the particle swarm algorithm to obtain the solution. The output distribution of optimization is computed by Monte Carlo simulation, thus theoretically verifying the feasibility of the robust optimization. Finally, a suite of experimental apparata for EMDs have been designed and constructed, and actual measurements have been performed to verify the robust optimization. The flow chart of the method is shown in Fig. 2.
Fig. 2 The flow chart of the robust design method

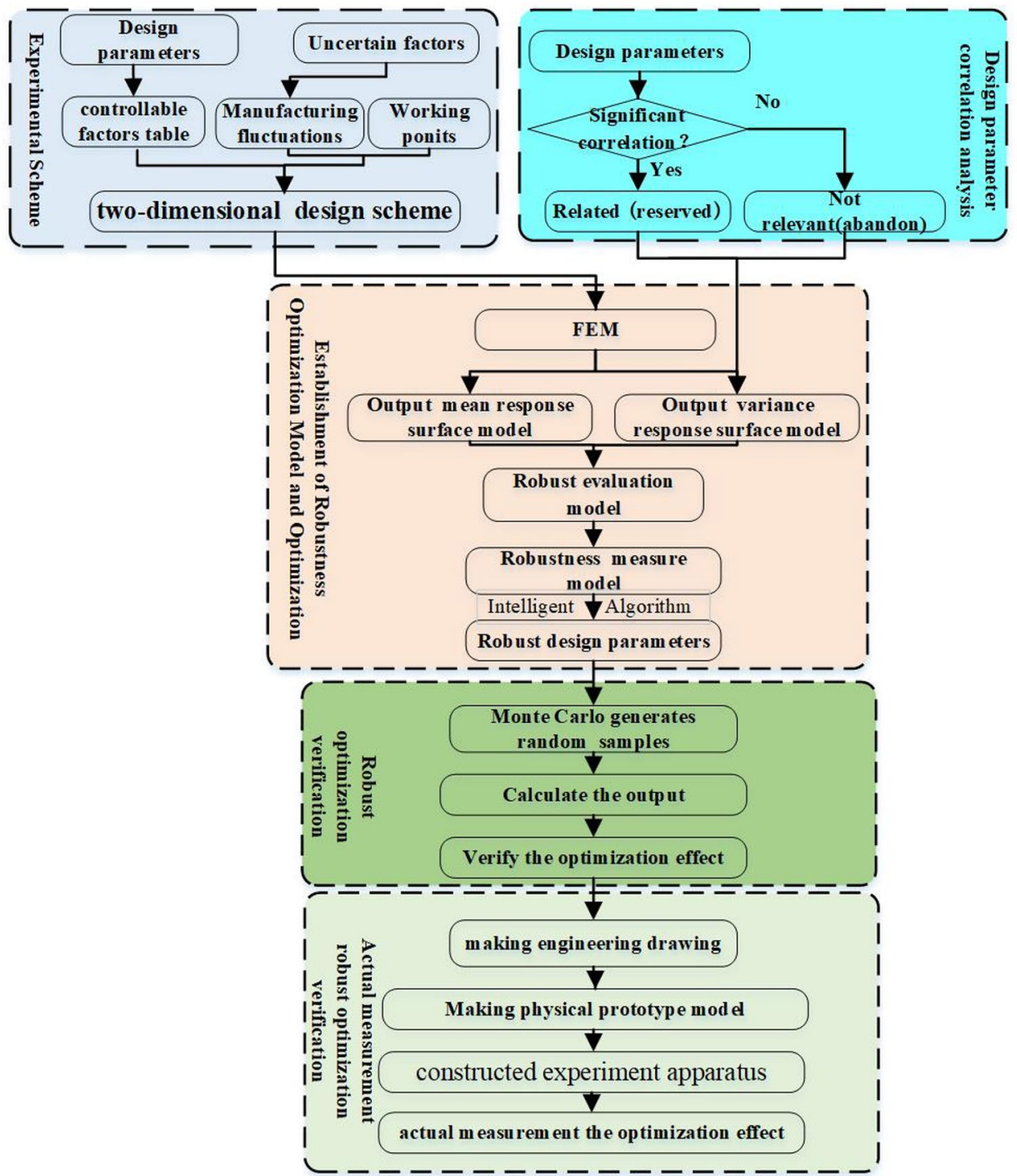

SN Applied Sciences A SPRINGER NATURE journal. 


\subsection{Experimental design considering,manufacturing uncertainties and the working point migration of permanent magnet}

Figure 3 presents the external surface design that considers the working point migration of the permanent magnets. The internal and external sampling are designed as follows: the internal sampling is determined by the experimental factors (design parameters), whereas the external one is designed using the manufacturing and working point migration factors. The design parameters $\mathrm{X}_{1}, \mathrm{X}_{2}, \ldots, \mathrm{X}_{m}$ are selected according to the research object and the robustness optimization target (in the inner table). Depending on the number of the parameters and levels, the Latin hypercube or uniform sampling method is adopted to determine the experimental scheme. The various uncertainties $\left(Y_{1}, Y_{2}, \ldots, Y_{n}\right)$ of the manufacture processing and operation environment are set by the current state of the manufacturing process. Their experimental samples may come from orthogonal experiments or Latin hypercube sampling, such that the EMDs have three manufacturing noise uncertainties, each with two levels. The manufacturing noise constitutes a $L 42^{3}$ orthogonal array. The permanent magnet working point of the EMDs is calculated from the load conditions. The working point migration factor has three levels, and the number of migrations may be enlarged according to the actual situation. Hence, the improved orthogonal array is used in the external design.

In order to enhance the effect of permanent magnet work point offset noise in the process of parameter design, this paper improves the traditional design of internal and external surface parameters to a certain extent. The noise factor mainly affects the appearance, and the noise forms the appearance in the form of orthogonal table. However, in this paper, in addition to the manufacturing noise, the influence of the permanent magnet work point offset noise should be considered, and a two-dimensional

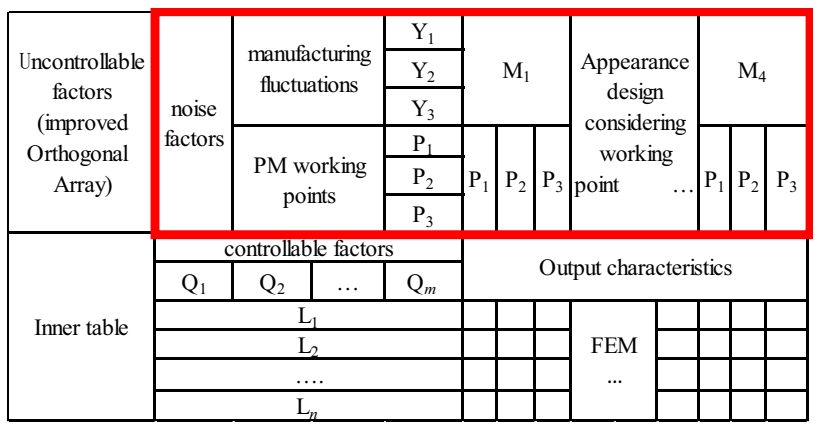

Fig. 3 Experimental design that considers the working point migration

SN Applied Sciences

APRINGER NATURE journa noise system should be established. Finally, the external surface has been modified, and the schematic diagram of the improved external header is shown in Fig. 3. In the example, there are three manufacturing noise factors, each of which is 2 levels, and the manufacturing noise constitutes the orthogonal table of $\mathrm{L}_{4} 2^{3}$; in the example, the deviation factor of permanent magnet working point is 3 levels, which adopts uniform design, and the number of temperature factors can be expanded according to the actual situation. In the example, there are three permanent magnet work point shift noise under each manufacturing noise, and the appearance is finally tested in a group.

\subsection{Correlation between design parameters and building of approximation model}

Non-repetitive combination $\left(a_{1}, a_{2}\right)$ of any two factors is taken from the experimental factors. The factors $x_{1}$ and $x_{2}$ are changed separately with the variation amounts $\Delta b_{1}$ and $\Delta b_{2}$ of output characteristics corresponding to the respective changes. Then, the variation amount $\Delta c$ of the output characteristics corresponding to the simultaneous change of $\left(a_{1}, a_{2}\right)$ is calculated. If the factors $a_{1}$ and $a_{2}$ are independent of each other, one may assume $\Delta c=\Delta b_{1}+\Delta b_{2}$.

An interaction factor $\gamma$ is introduced in Eq. (1). to take into account the degree of correlation between the factors $a_{1}$ and $a_{2}$. The response surface model is then built using the information contained in $\gamma$, i.e. correlated parameters are retained and theur cross terms are fully considered in creating the model, whereas uncorrelated or weakly correlated parameters are neglected.

$\left|\Delta c-\left(\Delta b_{1}+\Delta b_{2}\right)\right| \geq \gamma \times|\Delta c|$

In order to implement an optimization process with continuously changing factors, and accurate determination of the functional relationship between input parameters and output characteristics is needed. Response surface modelling of mean and variance allows us to properly include product performance and volatility characteristics, thus effectively improving robust design efficiency. The basic principle is illustrated in formulas (2) and (3), where the sums are the error terms of the mean and variance. The FEM is then performed on the experimental samples obtained by the sampling method, and the output characteristic parameters of EMDs are obtained. Based on the parameter correlation analysis, the high-order approximation models for the mean and variance between the design parameters and output characteristics is built using the high-order response surface model described in Eqs. (2) and (3). 


$$
\begin{aligned}
Y_{u}= & b_{0}+\sum_{i=1}^{k} b_{i} x_{i}+\sum_{i=1}^{k} b_{i i} x_{i i}^{2}+\cdots \\
& \cdots+\sum_{i=1}^{k} b_{i i} x_{i i}^{q}+\sum_{j k(j<k), l=1 \cdots q}^{k} b_{j k} x_{j}^{l} x_{k}^{q-l}+\varepsilon_{u} \\
Y_{\sigma}= & c_{0}+\sum_{i=1}^{k} c_{i} x_{i}+\sum_{i=1}^{k} c_{i i} x_{i i}^{2}+\cdots \\
& \cdots+\sum_{i=1}^{k} c_{i i} x_{i i}^{q}+\sum_{j k(j<k), l=1 \cdots q}^{k} c_{j k} x_{j}^{l} x_{k}^{q-l}+\varepsilon_{\sigma}
\end{aligned}
$$

\subsection{Building assessment optimization models}

Our high-order response surface model considers parameter interaction and it may improve the accuracy of parameter computation while enhancing, at least to some extent, the optimization efficiency of the robust optimal solution. On the other hand, the output characteristics of EMDs are determined by several factors including larger-the-better, smaller-the-better and nominally-the-best characteristics. Due to the lack of a robustness assessment method for unified evaluation of different output characteristics, the robustness optimal of the EMDs still remains a challenging process. In order to solve the problem, we introduce a robust assessment value of the different output characteristic of the target value. In particular, the various output characteristics are converted into the smaller-the-better characteristics, to facilitate multi-objective optimization, according to Eq. (4). The robust assessment value is defined by the target properties: $R$ is objective function of torque, $w_{1}$ is the weight of manufacturing factors, $w_{2}$ is the weight of the working point migration. The mean and variance simultaneously take into account the influence of manufacturing and working point migration of permanent magnets. In turn, the transformed robust assessment value is the smaller-the-better characteristic. It replaces the various output targets of the traditional robust design methods to achieve a unified robustness evaluation of different types of output characteristics.

$R_{i}= \begin{cases}e^{Y_{\mu}^{w_{1}}+Y_{\sigma}^{w_{2}}} & \text { smaller-the-best } \\ e^{\left(\left|Y_{\mu}^{w_{1}}-Y_{\text {expected }}\right|\right)^{w_{1}}+Y_{\sigma}^{w_{2}},}, & \text { nominal-the-best } \\ e^{Y_{\sigma}^{w_{2}}-Y_{\mu}^{w_{1}},} & \text { larger-the-best }\end{cases}$

\section{Case study (A type of polarized electromagnetic relay with permanent magnets used in electric vehicles)}

As a case study, we here consider a polarized electromagnetic relay with permanent magnets. The scheme is shown in Fig. 4. The working process is as follows: When the coil is not energized there is always a small air gap such that the armature of the permanent magnet in the EMD may pull. In this case, the moving reed (moving contact) in the contact spring system is in contact with the stator and the static contact. On the contrary, when the coil is energized, the static contact is disconnected and the armature is subjected to an electromagnetic force. Initially, the pusher is not in contact with the moving reed. After a period of time, the sheet contacts, making the moving reed to move. As a consequence, the movable contact and the static contact that are not in contact with each other are closed, and the suction process is completed. When the coil starts to be powered down, the electromagnetic system has a permanent small air gap to make the armature. The armature is held, and the force is greater than the reaction force provided by the spring system, and the armature is maintained. In the electromagnetic system, the following parameters are analyzed step by step: magnet gap yoke, pole surface distance, permanent magnet size, armature size, yoke size, small shaft size, core size, upper bracket size, lower bracket size, and push ball.

According to the electromagnetic system $\rightarrow$ magnetic gap yoke pole surface distance and armature coaxially $\rightarrow$ permanent magnet size parameters, armature size parameter, yoke size parameter, small shaft size parameter, core size parameter, upper bracket size parameter, lower bracket size parameter, and push ball size parameters are analyzed step by step, as shown in Fig. 5. and Table 1.

The main components and their main manufacturing process are shown in Fig. 5. Most of the components are mass-produced using modern production equipment. The parameters are of course approximate and subject

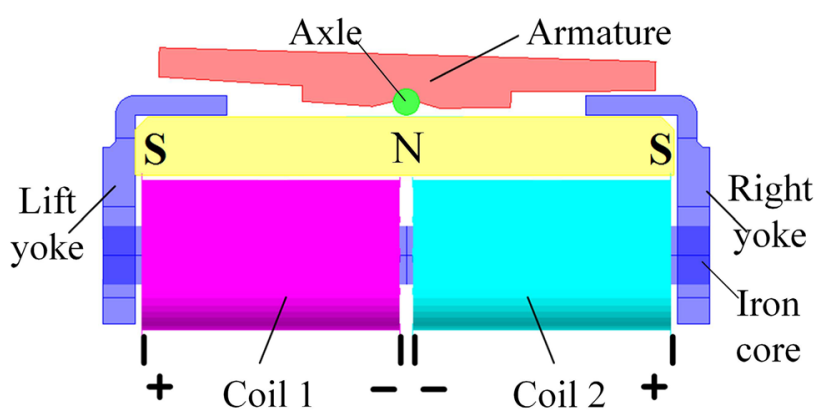

Fig. 4 EMD with double permanents 


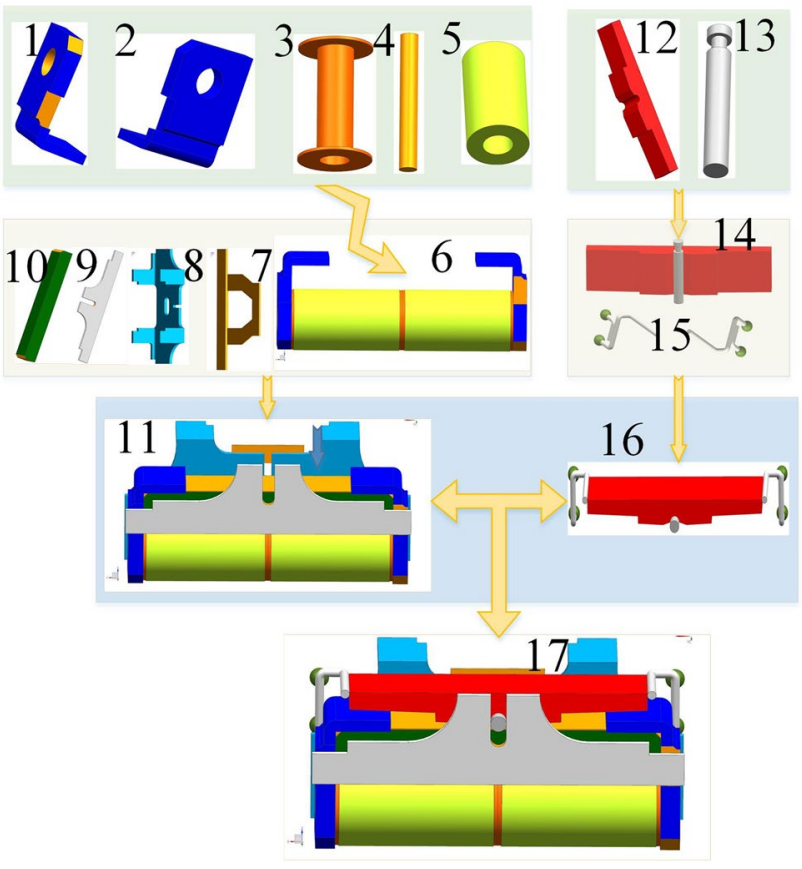

Fig. 5 EMD with double permanents manufacturing process

Table 1 Component name

\begin{tabular}{llcl}
\hline Number & Name & Number & Name \\
\hline 1 & Left Yoke & 2 & Right Yoke \\
3 & Coil Frame & 4 & Core \\
5 & Coil & 6 & $\begin{array}{l}\text { Electromagnet } \\
\text { Assembly }\end{array}$ \\
7 & Support Plate & 8 & Stent \\
9 & Front mounted & 10 & Permanent Magnet \\
& Toolbar Plate & & \\
11 & Yoke Component & 12 & Armature \\
13 & Small Shaft & 14 & Small Shaft \\
& Component \\
15 & Pushing Rod & 16 & Armature Component \\
17 & Electromagnetic & & \\
& system & & \\
\hline
\end{tabular}

to normal distribution. There are six key design parameters, which are $\gamma_{1}$ width of left and right yoke pole face, $\gamma_{2}$ thickness of permanent magnets, $\gamma_{3}$ width of yoke, $\gamma_{4}$ diameter of iron core, $\gamma_{5}$ width of armature and $\gamma_{6}$ length of armature. Most of its components are mass-produced using modern production equipment. The parameters are mostly approximate and subject to normal distribution. The main parameters are shown in Table 2 These six design parameters have five levels (see the inner table L25) such that $5^{6}$ experimental schemes may be generated by orthogonal experiments.
Table 2 Upper and lower limits of dimensional parameters

\begin{tabular}{lrrl}
\hline$\gamma(\mathrm{mm})$ & Min & Max & Variance \\
\hline$\gamma_{1}$ & 3.0 & 4.5 & 0.1 \\
$\gamma_{2}$ & 2.0 & 3.0 & 0.08 \\
$\gamma_{3}$ & 1.0 & 1.5 & 0.06 \\
$\gamma_{4}$ & 16.3 & 17.8 & 0.18 \\
$\gamma_{5}$ & 11.0 & 11.5 & 0.15 \\
$\gamma_{6}$ & 14.3 & 14.8 & 0.15
\end{tabular}

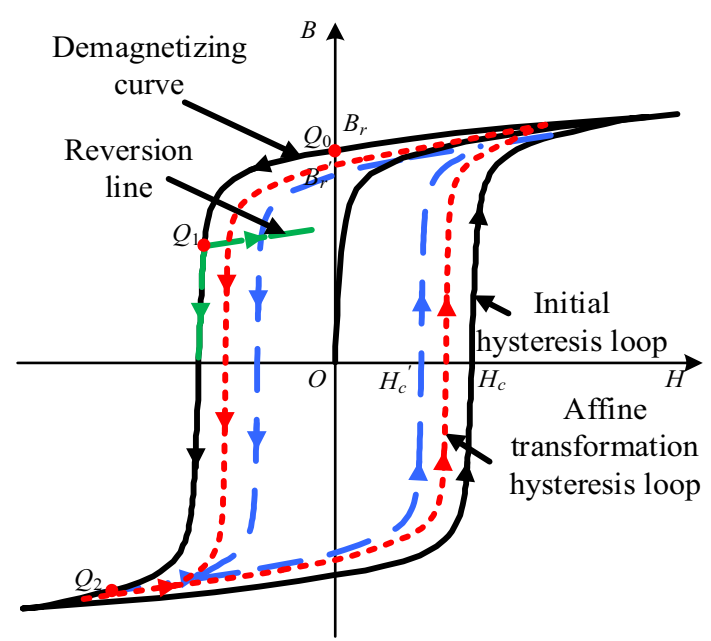

Fig. 6 Working point analysis

Upon applying an affine transformation to the main hysteresis loop, one may solve the unsaturated demagnetization hysteresis loop of the permanent magnet (as shown in Fig. 6 , blue and red dotted line). When the demagnetization hysteresis loop is not saturated, the current working point of the permanent magnet is in the fourth quadrant, the residual magnetization is $B r^{\prime}$, and the coercive force is $H c^{\prime}$. According to the demagnetization curve and the return line, if the permanent magnet is demagnetized by the external demagnetizing field, it migrates along the blue dotted line in Fig. 6 , near the $H$ axis. If the permanent magnet is magnetized, the line moves away from the $H$ axis. Combined with the demagnetization curve model and the return line model, the current working point of the permanent magnet and its working point change may be obtained.

According to the calculation method of permanent magnet working point shown in Fig. 6 , the calculated surface magnetic strengths of permanent magnet corresponding to the five horizontal permanent magnet working point migration points are $0.66 \mathrm{~T}, 0.68 \mathrm{~T}, 0.7 \mathrm{~T}, 0.72 \mathrm{~T}$ and $0.74 \mathrm{~T}$.

The noise factors are the following: $n_{1}$ width of armature, $n_{2}$ thickness of armature, $n_{3}$ length of iron core. These three factors have two levels and have formed four 
Table 3 Interactivity analysis results

\begin{tabular}{lllllll}
\hline & $\gamma_{1}$ & $\gamma_{2}$ & $\gamma_{3}$ & $\gamma_{4}$ & $\gamma_{5}$ & $\gamma_{6}$ \\
\hline$\gamma_{1}$ & - & - & - & - & - & - \\
$\gamma_{2}$ & 0.076 & - & - & - & - & - \\
$\gamma_{3}$ & 0.139 & -0.461 & - & - & - & - \\
$\gamma_{4}$ & 0.647 & 0.067 & 0.147 & - & - & - \\
$\gamma_{5}$ & 0.11 & 0.144 & 0.083 & 0.053 & - & - \\
$\gamma_{6}$ & 0.365 & 0.126 & 0.118 & 0.047 & 0.065 & - \\
\hline
\end{tabular}

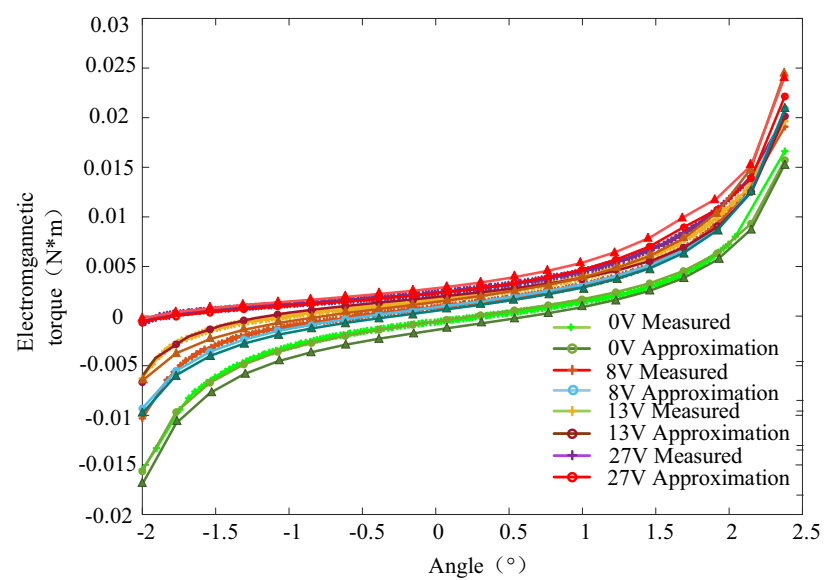

Fig. 7 Comparison between actual measurement and approximate model

experimental programs. The working points of the permanent magnets in the operating ampere-turn have five levels. Therefore, the noise factors and the working point of the permanent magnets, factored together, leads to 20 experimental schemes.

The output characteristics when the design parameters change, either individually or simultaneously, have been calculated, and the interactive analysis has been made according to the variation of the output characteristics. The results of this analysis are shown in Table 3.

As it is apparent from Table 3 the pairs $\left(\gamma_{1}, \gamma_{4}\right),\left(\gamma_{1}, \gamma_{6}\right)$ and $\left(\gamma_{2}, \gamma_{3}\right)$ show the strongest interaction (assuming the value 0.15 as a threshold). This evidence, combined with the two-dimensional experimental schemes, has been then employed to build the high-order response surface model (HIRSD) with interaction-model design parameters.

The approximate model results have been compared with the actual measurement results, and the state-of-art response surface model. Results as shown in Fig. 7. As one may expect, there are small discrepancies between HIRSD results and actual measurement results. The maximum error is about $6.7 \%$ in the initial position at $8 \mathrm{~V}$.

Figure 8 shows a group output of 750 characteristics, including the output mean and variance (combinations of different coil voltages and angles), of the contrasted

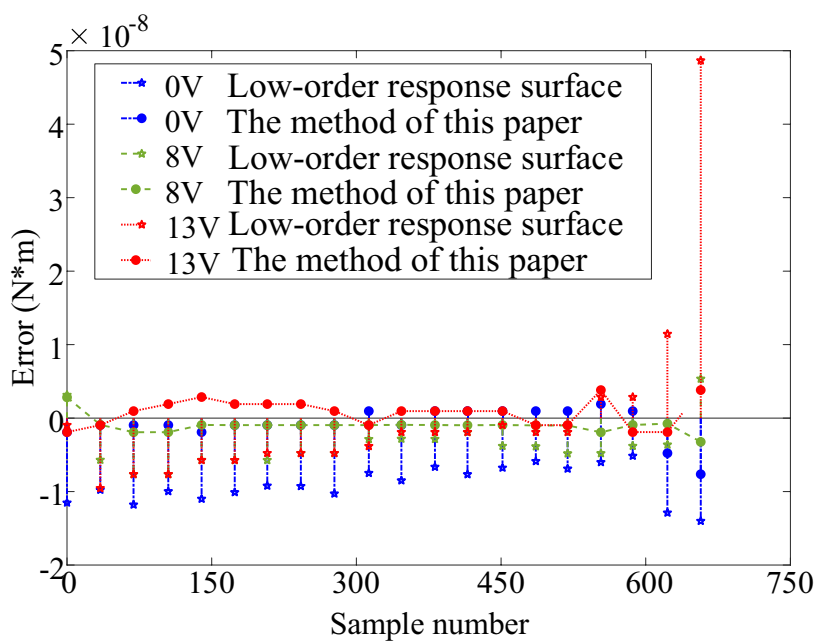

Fig. 8 Comparison between actual measurement and approximate model

condition between random test results and computed results of this research, with additional 15 prototypes. The results of this study indicate that errors are always smaller than those obtained with state-of-the-art response surface model.

$$
\left\{\begin{array}{l}
\operatorname{Min} R_{1}, R_{2} \\
3 \times 10^{-3} \leq \gamma_{1} \leq 4.5 \times 10^{-3} \\
2 \times 10^{-3} \leq \gamma_{2} \leq 3 \times 10^{-3} \\
1 \times 10^{-3} \leq \gamma_{3} \leq 1.5 \times 10^{-3} \\
16.3 \times 10^{-3} \leq \gamma_{4} \leq 17.8 \times 10^{-3} \\
11 \times 10^{-3} \leq \gamma_{5} \leq 11.5 \times 10^{-3} \\
14.3 \times 10^{-3} \leq \gamma_{6} \leq 14.8 \times 10^{-3}
\end{array}\right.
$$

Output values of the original design scheme have been obtained by HIRSM, and the elite set of particles has been obtained by Multi-Objective Particle Swarm Optimization. Particles with two objective-function output values, see Eq. (5) superior to the original design scheme are screened out from the elite set, and the non-dominated solution of design parameters is subsequently substituted into the objective function to gain the current Pareto optimal 


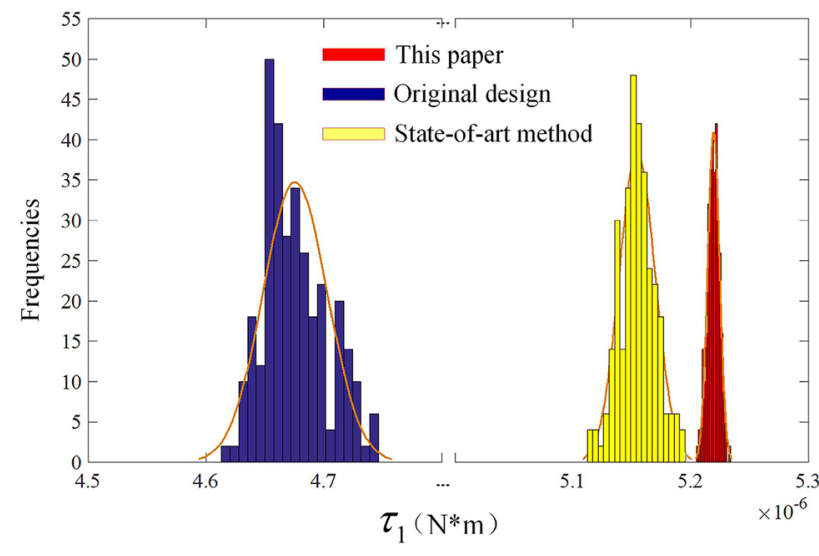

Fig. 9 Optimization results comparison at $0 \mathrm{~V}$

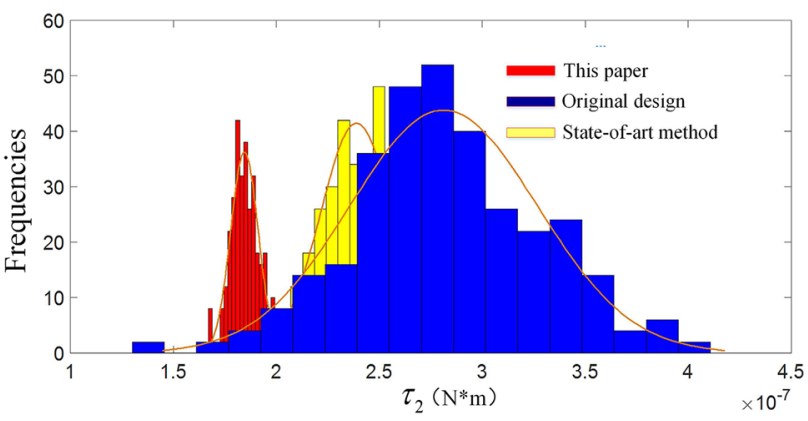

Fig. 10 Optimization results comparison at $13 \mathrm{~V}$

Table 4 Robust evaluation value optimization comparison

\begin{tabular}{lccccc}
\hline & \multicolumn{2}{l}{ SAM (rise rate) } & & \multicolumn{2}{l}{ NPM ( rise rate) } \\
\cline { 2 - 3 } \cline { 5 - 6 } & Mean & Robustness & & Mean & Robustness \\
\hline$\tau_{1}$ & $10.75 \%$ & $43.62 \%$ & & $12.5 \%$ & $65.37 \%$ \\
$\tau_{2}$ & $8.26 \%$ & $23.5 \%$ & & $34.5 \%$ & $78.5 \%$ \\
Compare & 0.86 & 0.74 & & 0.28 & 0.37 \\
RVM & $17.78 \%$ & & & $39.65 \%$ & \\
\hline
\end{tabular}

solution set. On reapeating this procedure, the optimized results eventually converge to the globally optimal solution. The method used in this paper, the orthogonal method, and the state-of-the-art method may be compared in Figs. 9, 10 and Table 4.

Figure 9 shows that the torque value $\tau_{1}$ in the initial position at $0 \mathrm{~V}$ influences the vibration performance of the relay, where the greater value is better. When the EMD is not powered, the working point of the permanent magnets does not migrate. At this time, the central value of the optimization result obtained by the state-of-the-art method is close to that obtained by our method, and both, the output quality and the robustness are improved considerably over the original design.

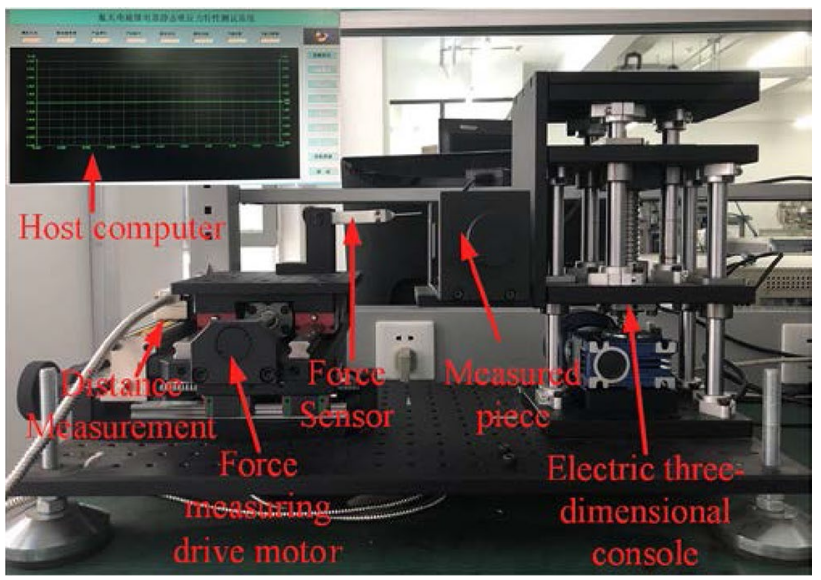

Fig. 11 Measured measurement system schematic

However, when the coil is powered, the working point of the permanent magnets migrate. The optimization result obtained by the state-of-art method greatly differs from the present method. The mean value, the robustness distribution and the results obtained with the present method are more reliable, with a small fluctuation of the output characteristics, as shown in Fig. 10. To further compare the optimization effects of our method to the state-of-the-art method (SAM), the effect of the traditional optimization method is calculated based on the optimization results of our method. Results are shown in Table 4.

Table 4 shows that before the coil is energized, the optimization effect of the traditional method can reach approximately $80 \%$ of our method. After the coil is powered, the optimization effect of the traditional method is only $30 \%$ of our method. At the same time, the robust evaluation value of the optimization method of this paper decreased from 0.82 to 0.39 , a decrease of $52.4 \%$, better than the state-of-the-art method of $64 \%$. On the one hand, the change of the permanent magnet working point during power-on is explained, and on the other hand, the effectiveness of the optimization method is verified.

At this point, 200 electromagnetic relays of this type were constructed using the optimization results. At the same time, we have designed and constructed a set actuators, including a Host computer, a Force sensor, an Electric three-dimensional console, a Distance measurement, and a Force measuring drive motor. The experimental apparatus is shown in Fig. 11. The torque measurement on the 200 electromagnetic relays has been carried out using the illustrated apparatus. Comparison of the experimental to the theoretical one is shown in Fig. 12.

The electromagnetic torque of the electromagnetic mechanism was measured at $0 \mathrm{~V}$ and $13 \mathrm{~V}$ in the initial position. As it is apparent from Fig. 12, the electromagnetic torque of the 200 products at $0 \mathrm{~V}$ is very close to the

\section{SN Applied Sciences}




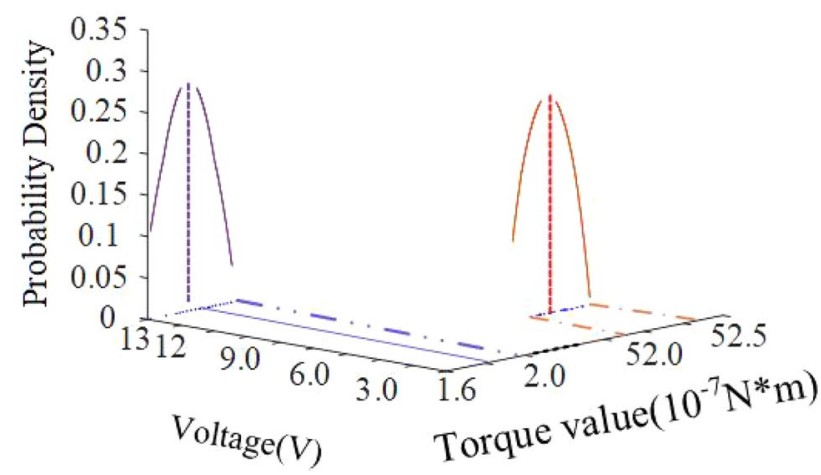

Fig. 12 Comparison of measured optimization results

theoretical value expected from the optimization results, which further validates the effectiveness of the proposed method. At the same time, Fig. 12 shows that the variation of the central value of the electromagnetic torque is similar to the theory when a different voltage is applied to the coil. In addition, the distribution of electromagnetic torque does not change significantly under different voltages, which proves the robustness of the proposed optimization scheme.

\section{Conclusion}

Among the factors that affect the optimization of electromagnetic devices, a relevant role is played by the effective consideration of the working point migration of permanent magnets, the precision of the approximation model, and the accurate evaluation of the robustness of design in robust optimization. In this study, aiming at facing the current existing problems of EMD optimization, we have introduced a two-dimensional noise method to properly take into consideration the correlations among parameters into the approximation model, and to improve the robust assessment value of different output characteristics. Our results may be summarized as follows:

1. We have introduced an internal and external sampling experiment method for EMD. The method in this study addresses the inadequacy of the previous optimization methods in two directions: (a) to effectively consider the influence of the PM working point migration on the optimization; (b) to solve the shortcomings of the traditional Taguchi robust design method. Correlation analysis has been performed on the design parameters based on the samples, and the high-order dual RSM for the output characteristic mean and variance has been built by using small samples, which improves the approximate modelling accuracy and efficiency.
2. We have designed a robust assessment value model, which may comprehensively consider different target types and may address the problem of multiple target types in the EMD optimization. This is a significant improvement in the robustness and optimization efficiency of EMD under current manufacturing conditions, which has resulted in an increase of the average suction torque at the initial position by $34.5 \%$ at the pull-in voltage.

3. Within the original design tolerance limits, 200 sets of virtual prototype samples were randomly generated. The $\tau_{1}$ volatility was reduced to $34.6 \%$. Furthermore, the $\tau_{2}$ volatility was reduced to $21.5 \%$, improving the robustness and reliability of the EMD considerably.

Funding This work was supported by the National Natural Science Foundation of China [Grant No. 61671172].

\section{Compliance with ethical standards}

Conflict of interest The authors declare that they have no conflict of interest.

\section{References}

1. Li H, Wang Q, He F, Zheng Y, Sun Y (2019) Design, numerical simulation, and experimental validation of a novel electromagnetic blank holding system for conventional drawing process. Int J Mach Tool Manuf 102:2183-2193. https://doi.org/10.1007/ s00170-018-03225-5

2. Dong X, Jianqiang Yu, Wang W, Zhang Z (2017) Robust design of magneto-rheological (MR) shock absorber considering temperature effects. Int J Mach Tool Manuf 90(11):1735-1747. https ://doi.org/10.1007/s00170-016-9480-9

3. Chen F, Liu GY (2017) Active damping of machine tool vibrations and cutting force measurement with a magnetic actuator. Int J Mach Tool Manuf 89(1-4):691-700. https://doi.org/10.1007/ s00170-016-9118-y

4. Sarin VP, Vinesh PV, Manoj M, Chandroth A, Pezholil M, Kesavath $\checkmark$ (2019) Experimental realization of electromagnetic toroidal excitation for microwave applications. SN Appl Sci 1(8):99-111. https://doi.org/10.1007/s42452-019-0980-8

5. Ming Ye, Gongye X, Yonggang L, Jingyu P (2018) Global optimization of emission and energy consumption for plug-in hybrid electric vehicle equipped with electric-mechanical continuously variable transmission. Int J Mach Tool Manuf 94(9-12):33893398. https://doi.org/10.1007/s00170-017-1229-6

6. Da Luz CMA, Tofoli FL, dos Santos Vicente P, Vicente EM (2018) Assessment of the ideality factor on the performance of photovoltaic modules. Energy Convers Manag 167:63-69. https://doi. org/10.1016/j.enconman.2018.04.084

7. Azizur Rahman M, Qin R (1997) A permanent magnet hysteresis hybrid synchronous motor for electric vehicles. IEEE Trans Ind Electron 44(1):1629-1642. https://doi.org/10.1109/41.557498

8. Castellanos JDA, Rajan HDV, Rohde AK, Denhof D, Freitag M (2019) Design and simulation of a control algorithm for 
peak-load shaving using vehicle to grid technology. SN Appl Sci 1(9):365-379. https://doi.org/10.1007/s42452-019-0999-x

9. Patterson D, Ren D, Spee C (1995) The design and development of an axial flux permanent magnet brushless DC motor for wheel drive in a solar powered vehicle. IEEE Stochastic optimization methods applied to microwave imaging: a review. IEEE Trans Ind Appl 31(5):2649-2663. https://doi.org/10.1109/28.464519

10. Tseng K-J, Chen GH (1997) Computer-aided design and analysis of direct-driven wheel motor drive. IEEE Trans Power Electr 12(3):649-661. https://doi.org/10.1109/63.575679

11. Liu ZJ, Vourdas A, Binns KJ (1993) Coupled thermo-electromagnetic analysis of electromagnetic devices with permanent magnets. J Phys D Appl Phys 43(4):1565-1568. https://doi. org/10.1088/0022-3727/26/5/014

12. Özkaya KY, Beyaz MI (2015) An investigation on the electromagnetic design optimization of rotary micromachines with doublelayer permanent magnets. Sens Actuators A 222:335-340. https ://doi.org/10.1016/j.sna.2014.12.025

13. Hafner M, Schoning M, Hameyer K (2010) Automated sizing of permanent magnet synchronous machines with respect to electromagnetic and thermal aspects. Int J Comput Math Electr Electron Eng 29(5):1205-1217. https://doi.org/10.1108/03321 641011061425

14. Batista LS, Campelo F, Guimaraes FG, Ramırez JA (2014) Ant colony optimization for the topological design of interior permanent magnet (IPM) machines. COMPEL: Int J Comput Math Electr Electron Eng 33(3):927-940. https://doi.org/10.1108/ COMPEL-08-2013-0285

15. Jan K, Sykulski (2008) New trends in optimization in electromagnetics. In IET 7th international conference on computation in electromagnetics, vol 17, pp 44-49

16. Huang Y, Chen B, Lv Z, Guo F, Huang C (2019) A cost-effective method for robust and anti-corrosive superhydrophobic coatings. SN Appl Sci 1(6):2375-2380. https://doi.org/10.1007/s4245 2-019-0620-3

17. Kawase Y, Yamaguchi T, Iwashita K, Kobayashi T, Suzuki K (2006) 3-D finite element analysis of dynamic characteristics of electromagnet with permanent magnets. IEEE Trans Magn 42(4):13391442. https://doi.org/10.1109/TMAG.2006.870966

18. Ibrahim H, Patruni JR (2019) Taguchi method-based optimization of extinguishing parameters for minimizing the extinction time of gaseous fires. SN Appl Sci 1(12):1337-1340. https://doi. org/10.1007/s42452-019-1642-6

19. Ouagued S, Amara Y, Barakat G (2016) Cogging force analysis of linear permanent magnet machines using a hybrid analytical model. IEEE Trans Magn 52(7):1-4. https://doi.org/10.1109/ TMAG.2016.2521825

20. Kuo C-C, Lin B-T, Wang W-T (2019) Optimization of microridge punch design for deep drawing process by using the fuzzy Taguchi method. Int J Mach Tool Manu 103(9-12):2903-2916. https ://doi.org/10.1007/s00170-019-03773-4

21. Sadeghifar M, Sedaghati R, Jomaa W, Songmene V (2018) Finite element analysis and response surface method for robust multi-performance optimization of radial turning of hard 300M steel. Int J Mach Tool Manu 94:2457-2474. https://doi. org/10.1007/s00170-017-1032-4

22. Qiu L, Zhang S, Wang Z, Xingxing Hu, Liu X (2019) A robust optimization design method for sheet metal roll forming and its application in roll forming circular cross-section pipe. Int J Mach Tool Manu 103:2903-2916. https://doi.org/10.1007/s00170-01903773-4

23. Amrhein M, Krein PT (2010) Induction machine modeling approach based on 3-D magnetic equivalent circuit framework. IEEE Trans Magn 25(2):339-347. https://doi.org/10.1109/ TEC.2010.2046998

24. Antoun CA, Würsch C, Köchli C, Perriard Y (2015) Validity tests of superposition principle based on forward model for electromagnetic induction scattering. IEEE Trans Magn 51(3):1-4. https ://doi.org/10.1109/TMAG.2014.2365476

25. Wang Z, Shuiyu Yu, Chen LY, Li Y (2018) Robust design for the lower extremity exoskeleton under a stochastic terrain by mimicking wolf pack behaviors. IEEE Access 6:30714-30725. https:// doi.org/10.1109/ACCESS.2018.2838091

26. Dorica M, Giannacopoulos DD (2006) Response surface space mapping for electromagnetic optimization. IEEE Trans Magn 42(4):1123-1126. https://doi.org/10.1109/TMAG.2006.872018

27. Hwang K-Y, Lin H, Rhyu S-H, Kwon B-I (2015) A study on the novel coefficient modeling for a skewed permanent magnet and overhang structure for optimal design of brushless DC motor. IEEE Trans Magn 48(5):1918-1923. https://doi.org/10.1109/ TMAG.2011.2176135

28. Li Y, Xiao S, Rotaru M, Sykulski JK (2016) A dual kriging approach with improved points selection algorithm for memory efficient surrogate optimization in electromagnetics. IEEE Trans Magn 52(3):1-4. https://doi.org/10.1109/TMAG.2015.2486522

29. Xiao S, Rotaru M, Sykulski JK (2013) Adaptive weighted expected improvement with rewards approach in kriging assisted electromagnetic design. IEEE Trans Magn 49(5):2057-2060. https:// doi.org/10.1109/TMAG.2013.2240662

30. Nassar Albunni M, Rischmuller V, Fritzsche T, Lohmann B (2009) Multiobjective optimization of the design of nonlinear electromagnetic systems using parametric reduced order models. IEEE Trans Magn 45(3):1474-1477. https://doi.org/10.1109/TMAG2 009.2012683

31. Xiaoyong Z, Zhengming S, Li Q, Zixuan X, Xuqian P (2016) Multiobjective optimization of an outer-rotor $V$-shaped permanent magnet flux switching motor based on multi-level design method. IEEE Trans Magn 52(10):1-8. https://doi.org/10.1109/ TMAG2016.2581767

Publisher's Note Springer Nature remains neutral with regard to jurisdictional claims in published maps and institutional affiliations. 